\title{
Real-Time Neural Signal Sensing and Spike Sorting System Using a Modified Zero-Crossing Feature with Highly Efficient Data Computation and Transmission
}

\author{
Sungjin Oh, Sungmin Han, ${ }^{1}$ and Inchan Youn ${ }^{*}$ \\ Center for Bionics, Korea Institute of Science and Technology, \\ 5, Hwarang-ro 14-gil, Seongbuk-gu, Seoul, 02792, Republic of Korea \\ ${ }^{1}$ Department of Biomedical Science, Korea University College of Medicine, \\ 73, Inchon-ro, Seongbuk-gu, Seoul, 02841, Republic of Korea
}

(Received April 27, 2016; accepted March 22, 2017)

Keywords: neural signal sensing, real-time spike sorting, zero-crossing feature, highly efficient data transmission, neural recording

Highly efficient data computation and transmission are necessary to implement the high-performance analysis of real-time neural signals. In a previous study, a zero-crossing feature (ZCF) was proposed as a computationally efficient spike feature that enabled enhanced data transmission efficiency. In this paper, a real-time neural signal sensing and spike sorting system using a modified ZCF is presented. In the system developed, the ZCF extraction algorithm is modified to improve spike sorting accuracy. The data transmission efficiency is highly enhanced by the use of a minimized data set size. The size of the data set is reduced by $90.7 \%$ as compared with the raw data transmission. In an in vivo experiment using spinal nerves of a Sprague-Dawley rat, neural signals were successfully detected and amplified with a maximum gain of $85.1 \mathrm{~dB}$ and a signal-to-noise ratio (SNR) of $19.7 \mathrm{~dB}$. This experimental result shows that the neural spikes were more accurately detected and classified with the modified ZCF extraction algorithm as compared with the original algorithm. From these results, it is expected that this system can be effectively used for high-performance real-time neural signal analysis.

\section{Introduction}

Real-time neural spike sorting provides substantial benefits in closed-loop neural recordings and stimulating systems because feedback on neuronal activities can be obtained in real time. ${ }^{(1-4)}$ In addition, real-time spike sorting is necessary to develop a high-performance brain-machine interface (BMI) where real-time movement prediction is used to control prosthetic devices. ${ }^{(5-8)}$ Recently developed multi-channel implantable neural recording devices also require real-time neural spike sorting. ${ }^{(9)}$

Recently, a CMOS technology-based high-density micro-electrode array (HDMEA) composed of more than four thousand recording electrodes was used to detect complicated neuronal activities. ${ }^{(10-12)}$ Because of the significantly increased number of recording electrodes, the amount of recorded data was also dramatically increased. Thus, highly efficient data processing must be

${ }^{*}$ Corresponding author: e-mail: iyoun@kist.re.kr http://dx.doi.org/10.18494/SAM.2017.1552 
implemented to analyze a large amount of data in real time. In other words, the computational effort should be reduced to extract neural spike features more efficiently. In addition, the size of transmitted data sets must be minimized to ensure fast data communication.

Traditional spike sorting algorithms, such as principal component analysis, template matching, and wavelet transform, are inappropriate to implement highly efficient real-time spike sorting because they require heavy computational effort and offline training to extract spike features. ${ }^{(13-17)}$ In a previous study, a zero-crossing feature (ZCF) was proposed as the computationally efficient spike classification element with a significantly reduced data set size. ${ }^{(18,19)}$ However, the original ZCF extraction algorithm can cause inaccurate sorting results because the number of samples in a temporal window for a spike is predetermined and fixed, regardless of the duration of the spike. ${ }^{(18)}$

In this paper, a real-time neural signal sensing and spike sorting system using the modified ZCF is presented. The system is composed of an analog neural amplifier with frequency filters, a fieldprogrammable gate array (FPGA)-based digital data processor to extract the ZCF, and a Matlabbased clustering software program operating in a PC. To enhance the spike sorting accuracy, the ZCF extraction algorithm is modified in the system. In the modified ZCF extraction algorithm, the number of samples in the temporal window for a spike is adjusted according to the duration of the spike.

The performance of the real-time neural spike sorting system was evaluated in an in vivo experiment using a Sprague-Dawley (SD) rat. In the experiment, neural spikes, obtained from dorsal root ganglions of the SD rat, were detected and sorted by the system.

\section{Methods}

\subsection{System architecture}

A system architecture of the real-time neural signal sensing and spike sorting system using the modified ZCF is shown in Fig. 1. The system consists largely of three parts: the analog neural amplifier with frequency filters, the data processor including an analog-to-digital converter (ADC), and the clustering software program. The neural amplifier carries out neural signal sensing, amplifying, and filtering processes. In the data processor, neural spikes and zero-crossing (ZC) points are detected from the acquired neural signal. In addition, ZCFs of the detected neural spikes are extracted. Then, information on the extracted features is transmitted to the clustering software for classification of the detected neural spikes.

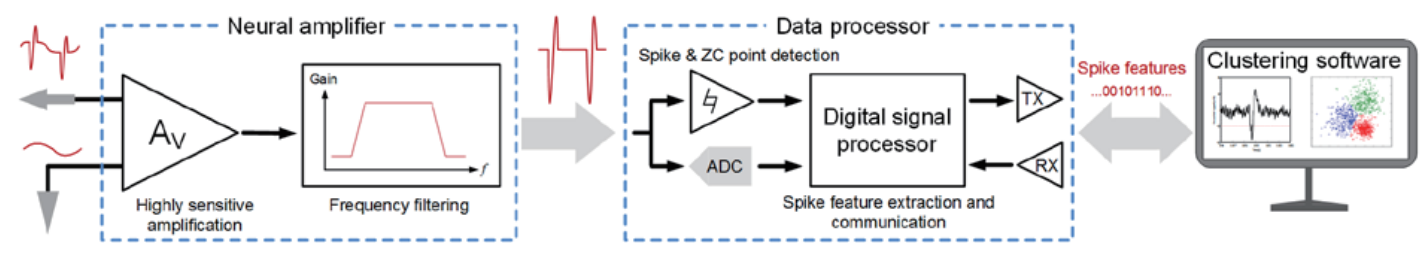

Fig. 1. (Color online) System architecture of the real-time neural signal sensing and spike sorting system using the modified ZCF. 


\subsection{Neural amplifier}

Signal amplification should be preferentially carried out in acquiring the neural signal because the amplitude of the signal is in the sub-mV range, generally less than $100 \mu \mathrm{V}$. To obtain sufficiently large amplitude of signal needed for postprocessing, the maximum amplification gain is set at $85 \mathrm{~dB}$. A differential operational amplifier (OP-AMP) is generally used to reject common noises from differential inputs that are connected to the recording electrode or reference electrode. In the OP-AMP, a sufficiently high input impedance of more than $100 \mathrm{M} \Omega$ is required to prevent signal attenuation caused by the potential divider effect. ${ }^{(20)}$ In addition, the high input impedance permits the use of a variety of electrodes because impedance matching becomes unnecessary. ${ }^{(21)}$ A high common mode rejection ratio (CMRR) is also required to reject the common mode interference that can cause significant distortion of the acquired signal. ${ }^{(22)}$ The input bias current of the amplifier circuit should be minimized because a very small amount of current can be provided from the nerves. ${ }^{(23)}$

In the acquired signal, large DC offsets associated with the electrodes, low-frequency fluctuation caused by the local field potential (LFP) (1 to $300 \mathrm{~Hz}$ ), and high-frequency instrumental noise (higher than $5 \mathrm{kHz}$ ) are mixed with neural spikes $(300 \mathrm{~Hz}$ to $5 \mathrm{kHz}$ ). Therefore, frequency filtering is necessary to separate the neural spikes from other signals. Before signal amplification, the DC offsets should be eliminated by an AC-coupling circuit. The LFP and high-frequency instrumental noise can be removed using high-pass and low-pass filters, respectively.

Figure 2 shows a schematic of the neural amplifier circuit designed according to the stated conditions. A two-stage gain amplifier topology, composed of a preamplifier and a noninverting gain amplifier, is used to obtain a sufficiently large amplification gain of $85 \mathrm{~dB}$. A gain programmable instrumentation amplifier integrated circuit (IC) (INA121; Texas Instruments Inc., Dallas, TX) is used as the preamplifier. The INA121 provides sufficiently high input impedance (159 $\mathrm{M} \Omega$ at $1 \mathrm{kHz})$, high CMRR $(98 \mathrm{~dB}$ at $1 \mathrm{kHz})$, and low input bias current $( \pm 4 \mathrm{pA})$. The preamplification gain is set at $40 \mathrm{~dB}$. AC-coupling capacitors are connected to the inputs of the preamplifier to remove the DC offsets associated with the electrodes.

At the filter stage and the second gain stage, a high-precision rail-to-rail OP-AMP (TLV2262; Texas Instruments Inc., Dallas, TX) is used. The LFP can be removed by a 2nd-order Sallen-

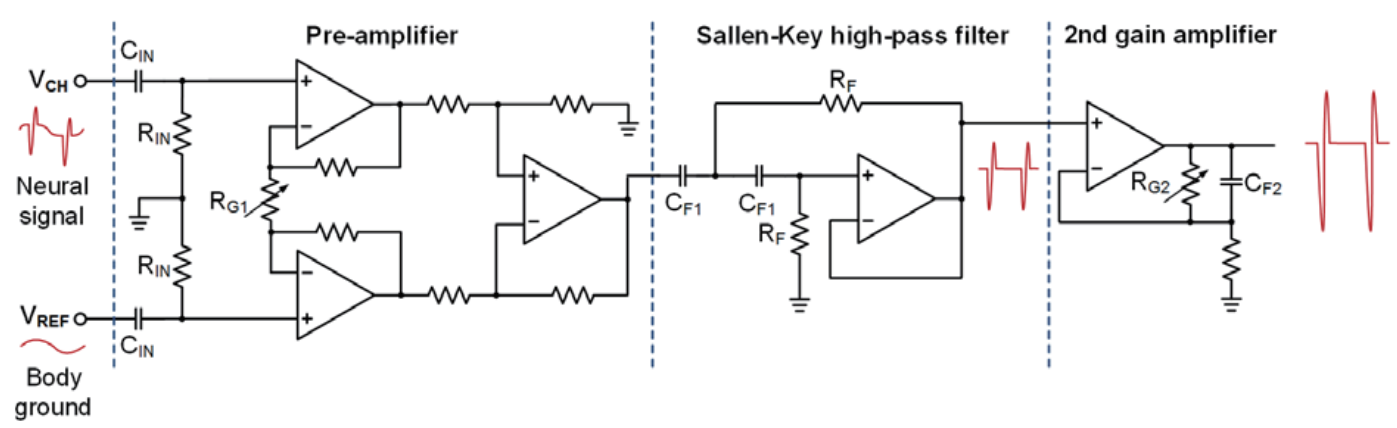

Fig. 2. (Color online) A schematic of the neural amplifier circuit consisting of two-stage gain amplifiers and noise-rejecting filters. 
Key high-pass filter with a cutoff frequency of $300 \mathrm{~Hz}$. After low-frequency suppression, the filtered signal is additionally amplified through the noninverting gain amplifier. Because a passive RC low-pass filter with a cutoff frequency of $5 \mathrm{kHz}$ is integrated in the feedback loop of the noninverting gain amplifier, the high frequency noise can also be removed.

The neural amplifier circuit is shown in Fig. 3. The neural amplifier is integrated in the printed circuit board (PCB) with an implantable size of $30 \times 12.5 \mathrm{~mm}^{2}$. At the head of the PCB, a nanostrip neuroconnector (A79039-001; Omnetics Connector Corp., Minneapolis, MN) is integrated to connect a variety of electrodes.

\subsection{Data processor}

In the data processor, the neural spike detection and feature extraction are carried out using an acquired neural signal. A schematic of the data processor is shown in Fig. 4. In a bias circuit, the amplitude and offset level of the acquired signal are modified to be appropriate for the signal processing conducted in the data processor. In addition, DC bias voltages used to detect spikes and $\mathrm{ZC}$ points are generated in the bias circuit. In a spike and ZC point detector, the neural spikes are

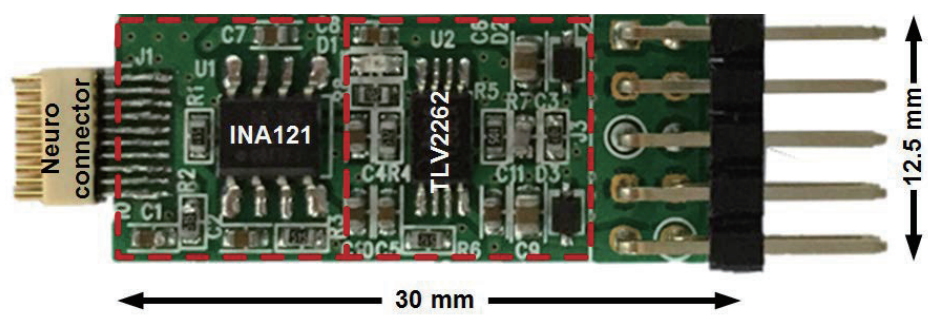

Fig. 3. (Color online) Neural amplifier circuit board including a neuroconnector for connection of the electrode. The neural amplifier is integrated with an implantable size of $30 \times 12.5 \mathrm{~mm}^{2}$.

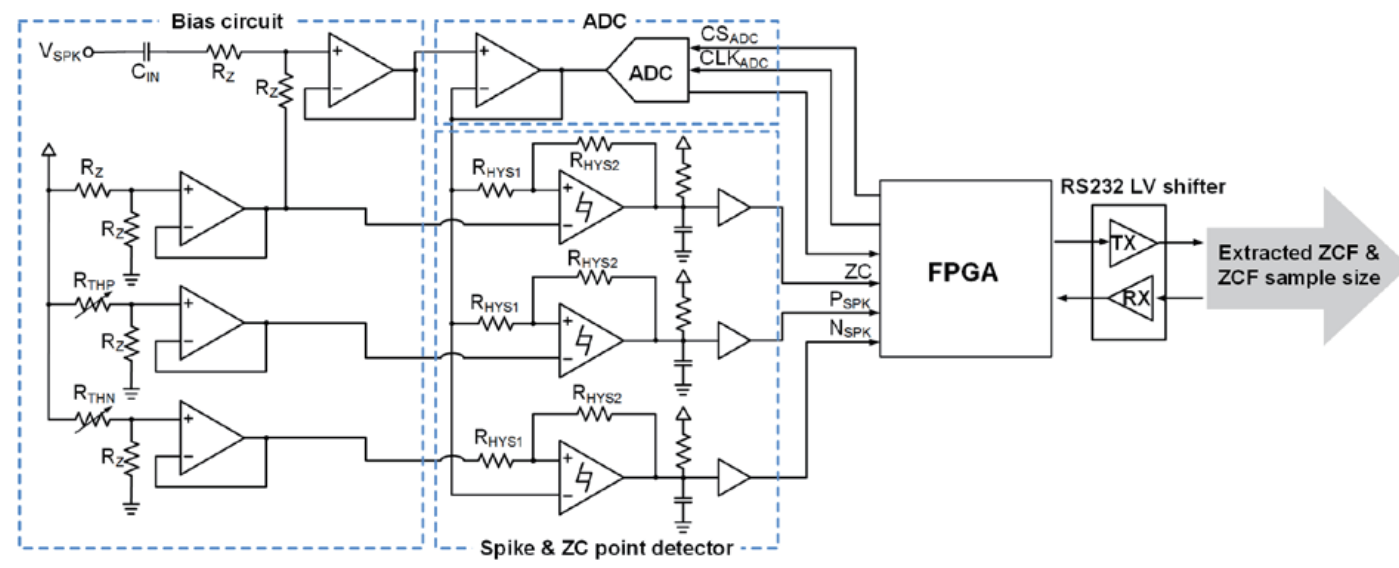

Fig. 4. (Color online) A schematic of the data processor circuit for dual-threshold spike detection, ZCF extraction, and data communication. The data processor is composed of a bias circuit, a spike and ZC point detector, an ADC, FPGA-based digital processor, and a data communication module. 
detected with dual thresholds. Spike detection accuracy can be improved by the dual-threshold method compared with that with the single-threshold method. ${ }^{(18)}$ The positive threshold $\left(V_{T H P}\right)$ and negative threshold $\left(V_{T H N}\right)$ levels are determined in the voltage dividers. Comparators (LM339; Texas Instruments Inc., Dallas, TX) with a hysteresis circuit are used to carry out low-noise spike detection based on the dual threshold method. Spike indicator signals $\left(P_{S P K}\right.$ and $\left.N_{S P K}\right)$ are determined according to the polarity of the detected spike.

The ADC (MAX11665; Maxim Integrated, San Jose, CA) converts the amplified analog neural signal to digital data with 12-bit resolution. The sampling rate is set at $50 \mathrm{kS} / \mathrm{s}$, which is sufficientry high to extract spike features accurately. In the FPGA chip (Xilinx Spartan-6 XC6SLX16; Xilinx Inc., San Jose, CA), two types of ZCFs, a pre-ZCF and a post-ZCF, are extracted at a clock frequency of $50 \mathrm{MHz}$. For more accurate feature extraction, a modified ZCF extraction algorithm is designed using the finite state machine (FSM)-based Verilog program. For each detected neural spike, $14 \mathrm{~B}$ of serial data including information on the extracted ZCFs and the number of samples constituting the ZCFs are generated and transmitted to the PC using RS-232 communication.

Figure 5 shows the data processor circuit. The data processor is integrated in the PCB with a portable size of $100 \times 80 \mathrm{~mm}^{2}$.

\subsection{Modified algorithm for ZCF extraction}

In Fig. 6, a sample plot of the acquired neural signal is shown to explain parameters used in the ZCF extraction algorithm. According to a previous study, the ZCF values can be calculated as

$$
Z C_{\text {Pre }}=\sum_{n=0}^{Z-1} x(n),
$$

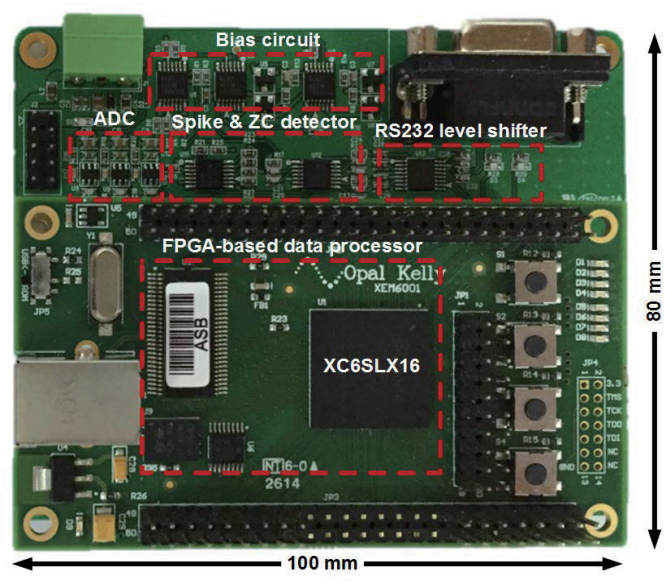

Fig. 5. (Color online) Data processor circuit board with a portable size of $100 \times 80 \mathrm{~mm}^{2}$.

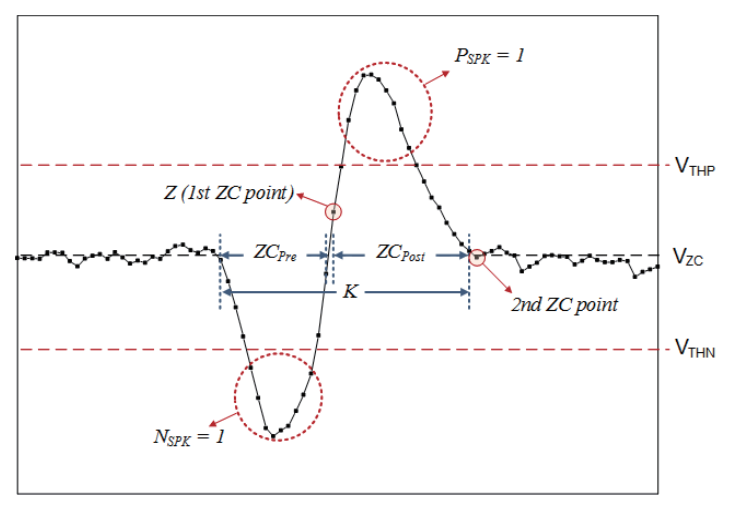

Fig. 6. (Color online) A sample plot of the acquired neural signal with parameters used for extracting the ZCF. 


$$
Z C_{\text {Post }}=\sum_{n=Z}^{K-1} x(n)
$$

where $Z$ is the index of the first $Z C$ time after a spike has been detected and $K$ is the number of samples in a temporal window for the spike. ${ }^{(18,19)}$

In a previous study, the number of samples in a spike, $K$ in Eq. (2), is defined as a predetermined value according to the sampling rate of the system. ${ }^{(18)}$ Therefore, the value of $K$ is fixed regardless of the real duration of the spike. This definition is valid when the spike duration is constant. In addition, a sufficiently long time interval between temporally adjacent spikes must also be guaranteed. However, even if neural spikes are detected at the same recording site, the duration of the spikes may vary depending on the neurons. ${ }^{(24)}$ In addition, the action potentials can burst in a nearly continuous fashion at very short time intervals because of multineuronal activities. ${ }^{(25,26)}$ Thus, post-ZCF values can be inaccurately extracted by the original algorithm presented in a previous study. ${ }^{(18)}$ In addition, the ZCFs of the temporally adjacent spikes at extremely short intervals can be significantly distorted. Thus, the value of $K$ must be automatically adjusted as a function of the spike duration to accurately extract the ZCFs.

In the system, a modified algorithm is used to extract the ZCFs from the acquired neural signals. In the modified ZCF extraction algorithm, the ZCFs are extracted until the second ZC point after spike detection. Therefore, the value of $K$ indicates the actual duration of the detected spike. This modification reduces the computational error in the post-ZCF extraction. In addition, the ZCFs can be accurately extracted even if the neural spikes burst nearly continuously with extremely short time intervals. The modified ZCF extraction algorithm is implemented by the FSM-based Verilog program. A state diagram of the FSM is shown in Fig. 7. The modified algorithm is simplified by using only four states.

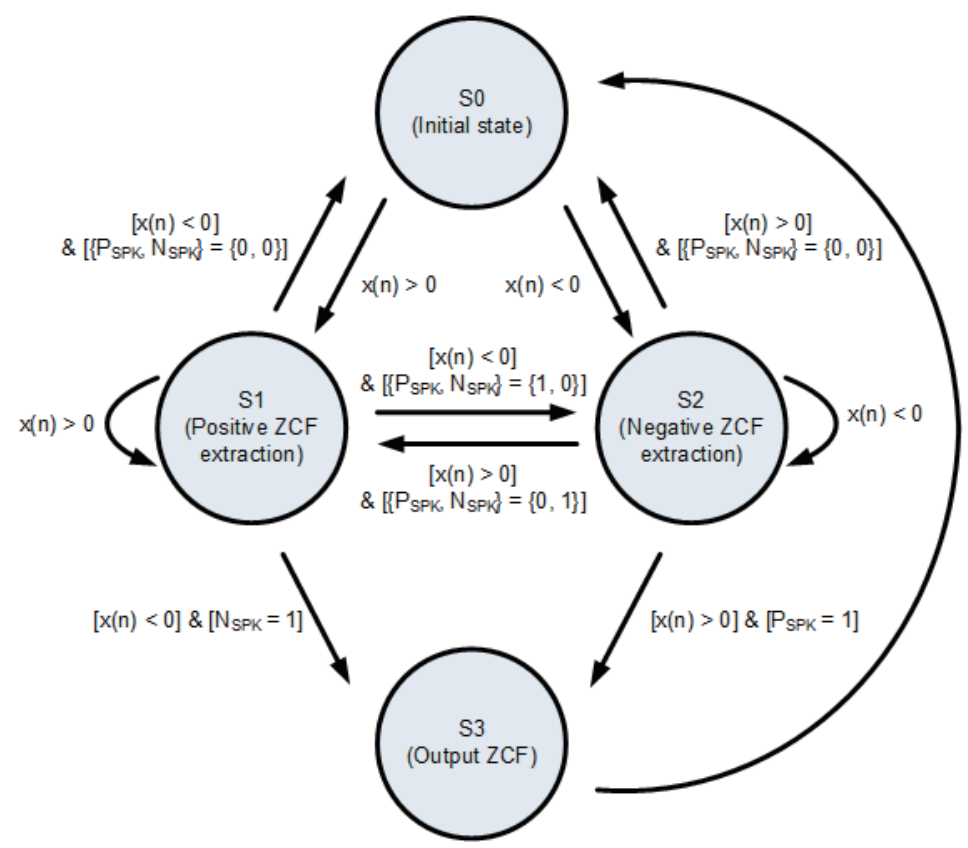

Fig. 7. (Color online) A state diagram of the FSM for the implementation of the modified ZCF extraction algorithm. The algorithm can be simplified as a total of four states. 


\subsection{Clustering software program}

The Matlab-based clustering software program was developed to classify the detected neural spikes according to the extracted ZCFs. In the clustering program, the $k$-means clustering method is used because of its computational simplicity. ${ }^{(27,28)}$ To optimize the number of clusters in $k$-means clustering, a silhouette coefficient that can evaluate cluster cohesion is used in the program. ${ }^{(29,30)}$

At first, $k$-means clustering is simultaneously carried out with a number of clusters from 2 to 8. Then, the silhouette coefficient, which means the average silhouette width of all elements, is calculated for each number of clusters. The silhouette coefficient is defined as

$$
S_{c o f f}=\frac{\sum_{i=1}^{N} s(i)}{N}=\frac{\sum_{i=1}^{N} \frac{b(i)-a(i)}{\max \{a(i), b(i)\}}}{N},
$$

where $s(i)$ is the silhouette width of the $i$ th element, $a(i)$ is the average distance of the $i$ th element to other elements in the same cluster, and $b(i)$ is the average distance of the $i$ th element to the elements in the nearest cluster. The number of clusters with the maximum silhouette coefficient is selected as the optimized number of groups for spike sorting.

\subsection{In vivo experiment}

An in vivo experiment was conducted to evaluate the performance of the real-time neural spike sensing and sorting system. All animal experimental procedures were approved by the Institutional Animal Care and Use Committee of the Korea Institute of Science and Technology. An adult male SD rat (body weight: $300 \mathrm{~g}$ ) was prepared for the experiment. The rat was anesthetized with an intraperitoneal injection of a solution containing ketamine $(36 \mathrm{mg} / \mathrm{kg})$ and xylazine $(8 \mathrm{mg} / \mathrm{kg})$ and located on a stereotaxic frame. Because compound action potentials can be easily and efficiently obtained at the spinal cord, the dorsal root ganglion in the lumbar vertebrae was determined as the recording site. Laminectomy surgery was conducted following the manner in our previous study. ${ }^{(31)}$ Briefly, the spinal cord and dorsal root ganglion between the third and sixth lumbar vertebrae were exposed by the laminectomy.

A needle-type parylene-C insulated tungsten microelectrode (A-M Systems, Sequim, WA) was used to acquire neural signals. The length and diameter of the needle electrode are $76.2 \mathrm{~mm}$ and $127 \mu \mathrm{m}$, respectively. The electrode impedance is $0.5 \mathrm{M} \Omega$ at $1 \mathrm{kHz}$, much smaller than the input impedance of the neural amplifier (159 M $\Omega$ at $1 \mathrm{kHz})$. The recording elecrode was connected to the neural amplifier through nanostrip neuroconnectors (A79038-001 and A79039-001; Omnetics Connector Corp., Minneapolis, MN) and inserted in the exposed dorsal root ganglion. The reference electrode, which was connected to the circuit ground signal, was positioned under the rat's skin around the spinal cord. In this experimental configuration, the body ground and circuit ground signals were connected to each other for noise reduction. Neural spikes from the dorsal root ganglion were acquired during a sensing time of $5 \mathrm{~s}$. The dual threshold of $\pm 500 \mathrm{mV}$ is used to detect neural spikes. The neural spikes were sorted in real time by the system. 


\section{Results}

\subsection{Electrical performance of the neural amplifier}

A plot of the frequency response of the neural amplifier is shown in Fig. 8. The neural amplifier provides the maximum amplification gain of $85.1 \mathrm{~dB}$ in a frequency region from $700 \mathrm{~Hz}$ to $2 \mathrm{kHz}$. Cutoff frequencies of the high-pass and low-pass filters are measured as $300 \mathrm{~Hz}$ and 5 $\mathrm{kHz}$, respectively. At a frequency region lower than $300 \mathrm{~Hz}$, the filter slope is measured as 23.9 $\mathrm{dB} / \mathrm{dec}$. The filter slope is measured as $-17.6 \mathrm{~dB} / \mathrm{dec}$ at the frequency region higher than $5 \mathrm{kHz}$.

\subsection{Neural signal acquisition and spike sorting}

The neural signal acquired in the in vivo experiment is plotted in Fig. 9. As shown in the plot, the neural spikes were clearly detected. In addition, the redundant signals, such as the DC offsets, LFPs, and high-frequency noises, were successfully removed. The signal-to-noise ratio (SNR) was $19.7 \mathrm{~dB}$.

Figure 10 shows the results of spike detection and classification. A total of 174 spikes were sorted in real time. Unclustered plots of the extracted ZCFs and detected spikes are shown in Figs. 10(a) and 10(b), respectively. The clustered results of the detected neural spikes are plotted in Figs. 10 (c) and 10(d). As shown in the color charts, the detected neural spikes were well classified into two groups. To compare the modified ZCF extraction algorithm to the original one presented in Ref. 18, spike detecting and sorting results using the original algorithm are also plotted in Fig. 11.

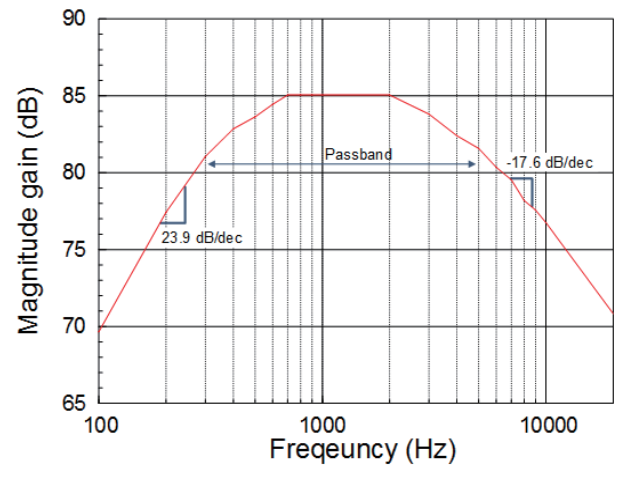

Fig. 8. (Color online) Measured frequency response of the neural amplifier. A maximum amplification gain of $85.1 \mathrm{~dB}$ and a passband of $300 \mathrm{~Hz}$ to $5 \mathrm{kHz}$ were obtained.

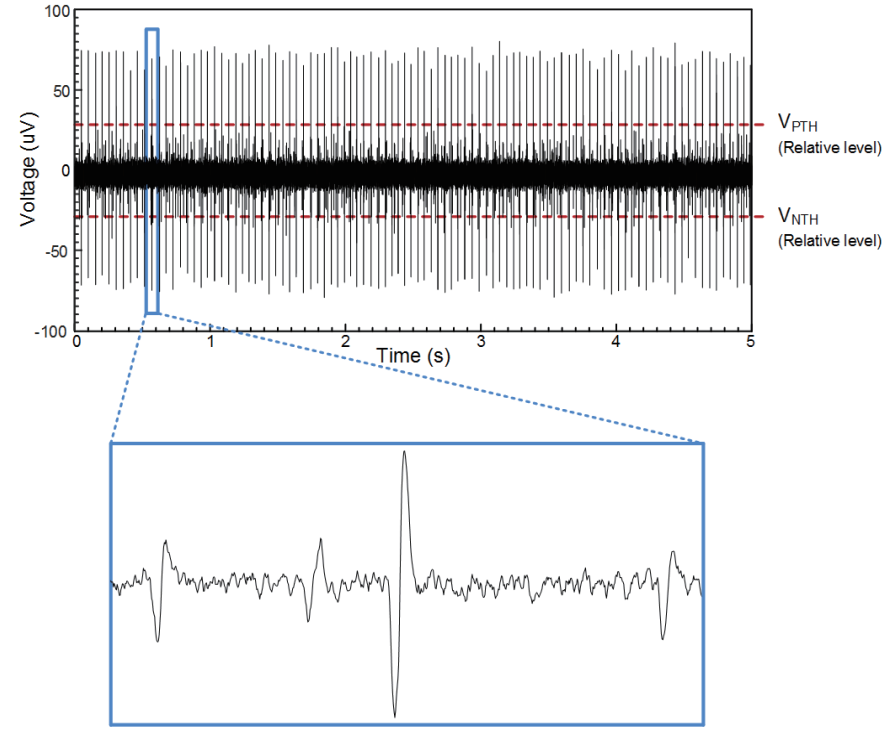

Fig. 9. (Color online) Acquired neural signal in the in vivo experiment. The neural spikes were clearly detected, and the noise signals were successfully removed. 


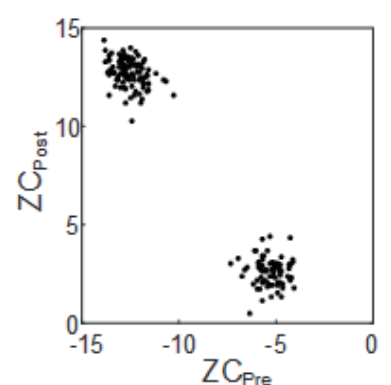

(a)

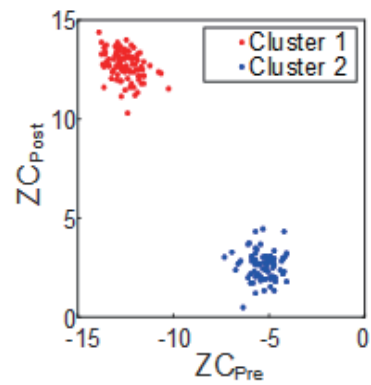

(c)

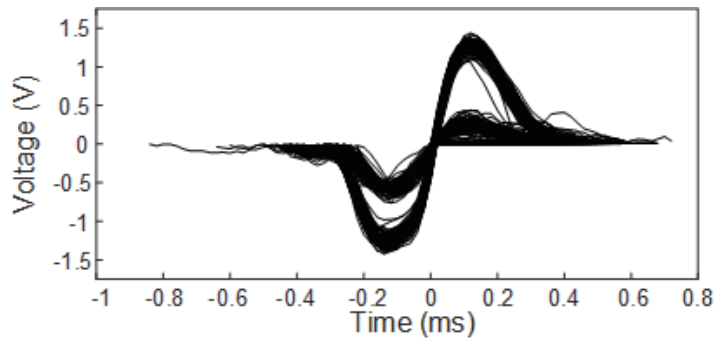

(b)

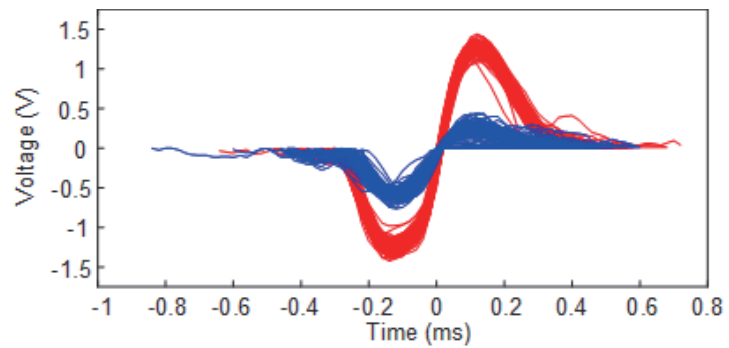

(d)

Fig. 10. (Color online) Neural spike detecting and sorting results using the system. (a) 2-D mapping of the extracted ZCFs, (b) temporal diagrams of the detected spikes, (c) clusters classified by the $k$-means clustering method, and (d) color-coded spikes in each cluster.

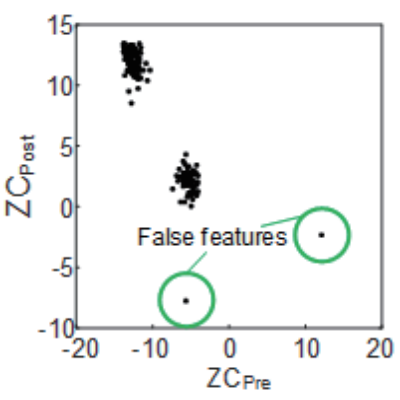

(a)

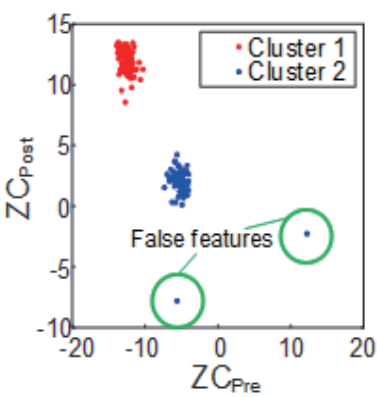

(c)

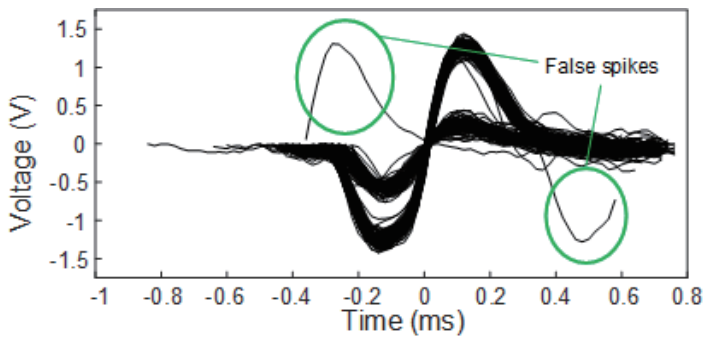

(b)

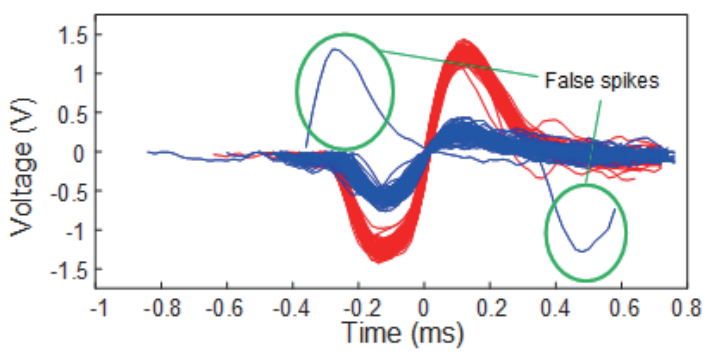

(d)

Fig. 11. (Color online) Neural spike detecting and sorting results based on the original ZCF extraction algorithm. (a) 2-D mapping of the extracted ZCFs, (b) temporal diagrams of the detected spikes, (c) clusters classified by the $k$-means clustering method, and (d) color-coded spikes in each cluster. 


\section{Discussion}

\subsection{Neural signal acquisition}

The neural signal was successfully detected in the in vivo experiment. As shown in Fig. 9, the neural spikes were clearly detected from the raw signal in which other signal elements, such as the DC offsets, LFPs, and high-frequency noises, were mixed with the neural spikes. Attributed to the high amplification gain of $85.1 \mathrm{~dB}$, the neural spikes with an amplitude less than $100 \mu \mathrm{V}$ were amplified to large-amplitude signals that are usable in the following processes. The noise elements were sufficiently rejected with an SNR of $19.7 \mathrm{~dB}$.

For evaluating the noise rejection performance of the system, frequency domain analysis of the acquired neural signal was conducted using the fast Fourier transform (FFT). Figure 12 shows the single-side amplitude spectrum and power density spectrum of the neural signal. As shown in the frequency spectra, noise signals with a frequency lower than $300 \mathrm{~Hz}$ or higher than $5 \mathrm{kHz}$, which are considered the nonspike signals including the DC offsets, LFPs, and high-frequency instrumental noise, were successfully rejected by the system.

\subsection{Spike sorting accuracy}

In the system, a modified algorithm is used to extract the ZCFs from the neural spikes. The in vivo experimental results on spike sorting show that the neural spikes were successfully grouped without the misclassification of signals. Thus, it can be inferred that the ZCFs were accurately extracted without computational errors. To compare the modified ZCF extraction algorithm with the original one, which was presented in the previous study, the acquired neural spikes were additionally sorted according to the method in Ref. 18. As shown in Fig. 11, with the original algorithm, some false signals were considered as neural spikes. In addition, the erroneous clustering result was caused because of the features of the false spike. From these results, it can be postulated that the modification in the feature extracting algorithm improves the accuracy of the spike sorting. In the modified ZCF extraction algorithm, the temporal window to extract ZCFs

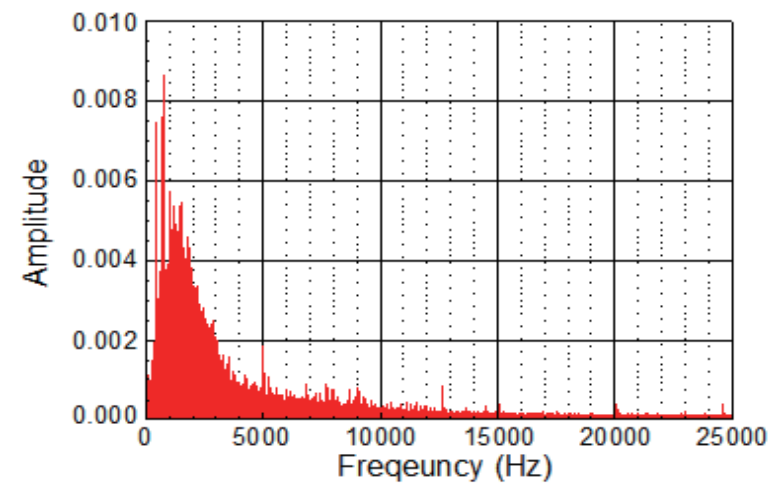

(a)

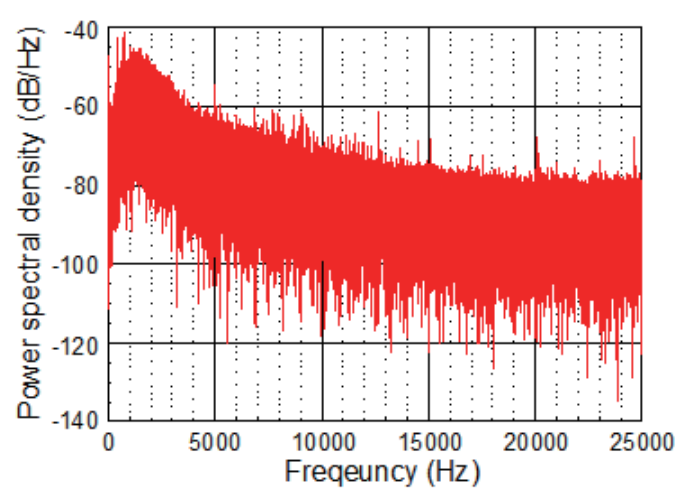

(b)

Fig. 12. (Color online) Frequency domain analysis of the neural signal acquired by the system in the in vivo experiment. (a) Single-side amplitude spectrum and (b) power density spectrum of the neural signal. 
from a spike is automatically adjusted to be equal to the spike duration to improve the accuracy of feature extraction.

\subsection{Data transmission efficiency}

Because of the minimized size of the data set, the real-time neural signal sensing and spike sorting system can provide highly efficient data transmission. For example, at least $150 \mathrm{~B}$ of data are required to transmit the waveform of a raw signal of a neural spike whose duration is 1 ms with the same sampling rate and data packet structure used in this new system. On the other hand, in the new system, only $14 \mathrm{~B}$ of data are necessary to transmit the ZCFs of a neural spike regardless of its duration. In other words, compared with the raw data transmission, the size of the transmitted data set for a neural spike is decreased by $90.7 \%$ in the developed system.

Enhancement of the transmission efficiency in the system can be more clearly shown with a continuously acquired neural signal. Unlike raw data transmission, information on the nonspike signals that occupy a large portion of the entire signal is selectively removed. For example, in the in vivo experiment, $2.44 \mathrm{kB}$ of data were used for transmitting the ZCFs of the neural spikes that were detected during the sensing time of $5 \mathrm{~s}$. On the other hand, the size of data for transmitting a waveform of the raw signal with a $5 \mathrm{~s}$ duration is calculated as $750 \mathrm{kB}$, which is approximately 300 times higher than the data size used in the experiment. This notable reduction in the data size can provide significant improvement in data transmission efficiency.

\section{Conclusions}

A real-time neural signal sensing and spike sorting system using a modified ZCF is described in this paper. By using ZCF for feature extraction, the data computation and transmission efficiencies can be greatly improved when compared with the transmission of raw data. In addition, spike sorting accuracy is enhanced because the ZCF extraction algorithm was modified. The in vivo experimental results show that the neural spikes were successfully detected and amplified with a maximum amplification gain of $85.1 \mathrm{~dB}$ and a SNR of $19.7 \mathrm{~dB}$. The spike sorting process was also successfully carried out in real time without any obvious errors. The spike sorting results indicate that the neural spikes were more accurately detected and classified by the modified ZCF extraction algorithm than by the original algorithm. ${ }^{(18)}$ In the system, the size of transmitted data for a spike is decreased by $90.7 \%$ when compared with the transmission of raw data. From these results, it is expected that the developed system can be effectively used for high-performance real-time neural signal sensing and spike sorting with highly efficient data computation and transmission.

\section{Acknowledgments}

This research was partially supported by a grant from the Korea Health Technology R\&D Project through the Korea Health Industry Development Institute (KHIDI), funded by the Ministry of Health \& Welfare, Republic of Korea (HI14C3477), and by the Next-generation Medical Device Development Program for Newly Created Market of the National Research Foundation (NRF), funded by the Ministry of Science, ICT and Future Planning, Republic of Korea (2015M3D5A1066100). 


\section{References}

1 K. S. Guillory and R. A. Normann: J. Neurosci. Methods 91 (1999) 21.

2 U. Rutishauser, E. M. Schuman, and A. N. Mamelak: J. Neurosci. Methods 154 (2006) 204.

3 F. Franke, M. Natora, C. Boucsein, M. H. J. Munk, and K. Obermayer: J. Comput. Neurosci. 29 (2010) 127.

4 F. Franke, D. Jackel, J. Dragas, J. Muller, M. Radivojevic, D. Bakkum, and A. Hierlemann: Front. Neural Circuits 6 (2012) 105.

5 D. M. Taylor, S. I. H. Tillery, and A. B. Schwartz: Science 296 (2002) 1829.

6 J. M. Carmena, M. A. Lebedev, R. E. Crist, J. E. O’Doherty, D. M. Santucci, D. F. Dimitov, P. G. Patil, C. S. Henriquez, and M. A. L. Nicolelis: PLoS Biol. 1 (2003) 193.

7 I. Obeid and P. D. Wolf: IEEE Trans. Biomed. Eng. 51 (2004) 905.

8 M. Alam, X. Chen, Z. Zhang, Y. Li, and J. He: PLoS One 9 (2014) e103764.

9 S. Gibson, J. W. Judy, and D. Markovic: Proc. 2008 30th Annu. Int. Conf. IEEE Eng. Med. Biol. Soc., eds. R. Buetra and R. Jones (IEEE, New York, 2008) pp. 5015-5020.

10 L. Berdondini, K. Imfeld, A. Maccione, M. Tedesco, S. Neukom, M. Koudelka-Hep, and S. Martinoia: Lab Chip 9 (2009) 2644.

11 U. Frey, J. Sedivy, F. Heer, R. Pedron, M. Ballini, J. Mueller, D. Bakkum, S. Hafizovic, F. D. Faraci, F. Greve, K. Kirstein, and A. Hierlemann: IEEE J. Solid-State Circuits 45 (2010) 467.

12 A. Lambacher, V. Vitzthum, R. Zeitler, M. Eickenscheidt, B. Eversmann, R. Thewes, and P. Fromherz: Appl. Phys. A 102 (2011) 1.

13 M. Abeles and M. H. Goldstein Jr.: Proc. IEEE 65 (1977) 762.

14 R. Q. Quiroga, Z. Nadasdy, and Y. Ben-Shaul: Neural Comput. 16 (2004) 1661.

15 Z. S. Zumsteg, C. Kemere, S. O’Driscoll, G. Santhanam, R. E. Ahmed, K. V. Shenov, and T. H. Meng: IEEE Trans. Neural Syst. Rehabil. Eng. 13 (2005) 272.

16 C. Vargas-Irwin and J. P. Donoghue: J. Neurosci. Methods 164 (2007) 1.

17 P. H. Thakur, H. Lu, S. S. Hsiao, and K. O. Johnson: J. Neurosci. Methods 162 (2007) 364.

18 A. M. Kamboh and A. J. Mason: IEEE Trans. Neural Syst. Rehabil. Eng. 21 (2013) 1.

19 A. M. Kamboh and A. J. Mason: Proc. 2010 Biomed. Circuits Syst. Conf., eds. T. Prodromakis and W. A. Serdijn (IEEE, New York, 2010) pp. 13-16.

20 A. C. M. van Rijn, A. Paper, and C. A. Grimbergen: Med. Biol. Eng. Comput. 28 (1990) 389.

21 B. R. Land, R. A. Wyttenbach, and B. R. Johnson: J. Neurosci. Methods 106 (2001) 47.

22 R. Pallas-Areny and J. G. Webster: IEEE Trans. Instrum. Meas. 40 (1991) 669.

23 T. Ativanichayaphong, J. W. He, C. E. Hagains, Y. B. Peng, and J. Chiao: J. Neurosci. Methods 170 (2008) 25.

24 C. W. Bourque and L. P. Renaud: J. Physiol. 363 (1985) 429.

25 G. E. Hardingham, Y. Fukunaga, and H. Bading: Nat. Neurosci. 5 (2002) 405.

26 C. P. J. de Kock and B. Sakmann: J. Physiol. 586 (2008) 3353.

27 H. Park and C. Jun: Expert Syst. Appl. 36 (2009) 3336.

28 A. K. Jain: Pattern Recognit. Lett. 31 (2010) 651.

29 P. J. Rousseeuw: J. Comput. Appl. Math. 20 (1987) 53.

30 G. Chen, S. A. Jaradat, N. Banerjee, T. S. Tanaka, M. S. H. Ko, and M. Q. Zhang: Statistica Sinica 12 (2002) 241.

31 S. Han, J. Chu, H. Kim, K. Choi, J. Park, and I. Youn: IEEE Trans. Biomed. Eng. 63 (2016) 1310. 